\title{
UMA ANÁLISE UNIFICADA PARA SUJEITOS INOVADORES (NULOS E MANIFESTOS) NA GRAMÁTICA DO PORTUGUÊS BRASILEIRO
}

\section{AN UNIFIED ANALYSIS FOR (NULL AND OVERT) INNOVATIVE SUBJECTS IN THE GRAMMAR OF BRAZILIAN PORTUGUESE}

Eloisa Nascimento Silva Pilati Universidade de Brasília Brasília, Distrito Federal, Brasil

Rozana Reigota Naves Universidade de Brasília Brasília, Distrito Federal, Brasil

Heloisa Maria Moreira Lima Salles Universidade de Brasília Brasília, Distrito Federal, Brasil

RESUMO: Este artigo tem dois objetivos principais: (I) descrever e analisar o licenciamento de sujeitos inovadores (nulos e manifestos) no Português Brasileiro (PB) sob o ponto de vista sincrônico, considerando a proposta de que a realização do sujeito é crucialmente determinada pela perda do traço de referencialidade na terceira pessoa, o que resulta na cisão do sistema pronominal/flexional (cf. PILATI e NAVES, 2011, 2013; PILATI, NAVES e SALLES, 2017a, 2017b); (II) apresentar dados da diacronia do sujeito no português brasileiro (PB), em comparação com o português europeu (PE) (cf. GRAVINA, 2014), como evidência para a cisão no paradigma pronominal/flexional do PB (cf. RABELO, 2010) e para a manifestação da ordem VS (verbo-sujeito) como inversão locativa nessa língua (cf. PILATI, 2006). Nesta abordagem, a categoria pronominal/flexional do PB na terceira pessoa é realizada como um feixe de traços $p h i$ (cf. HOLMBERG, 2010), sendo a realização de um DP (sintagma determinante o do inglês, determiner phrase) locativo (lexical ou nulo) na posição de sujeito determinada pela ausência do traço de referencialidade (cf. PILATI, NAVES e SALLES, 2017c).

PALAVRAS-CHAVE: Sujeitos nulos e manifestos; Português brasileiro (PB); Cisão do sistema pronominal/ flexional; Categoria dêitica (locativa/temporal) 
ABSTRACT: In this paper we have two goals: (I) to describe and to analyze the licensing of null and overt subjects in Brazilian Portuguese (BP) under a synchronic perspective, considering the proposal according to which the realization of the subject is crucially determined by the loss of the referential property in the third person of the pronominal/inflectional system (cf. PILATI e NAVES, 2011, 2013; PILATI, NAVES e SALLES, 2017a, 2017b); (II) to present diachronic data about the expression of the subject in Brazilian Portuguese (BP), in comparison with European Portuguese (EP) (cf. GRAVINA, 2014), as an evidence for the split in the pronominal/inflectional paradigm of BP (cf. RABELO, 2010) and for the analysis to the VS word order as locative inversion in this language (cf. PILATI, 2006). In this approach, in BP, the pronominal/inflectional category in the third person is realized as a cluster of phi features (cf. HOLMBERG, 2010), the presence of a locative DP (either lexical or null) in subject position being determined by the absence of the referential feature (cf. PILATI, NAVES e SALLES, 2017c).

KEYWORDS: Null and overt subjects; Brazilian Portuguese (BP); Split on the pronominal/inflectional system; Deictic (locative/temporal) category 


\section{INTRODUÇÃO}

Este artigo aborda um conjunto de construções do Português Brasileiro (PB) em que a sintaxe do sujeito é considerada inovadora no sentido de que não segue as propriedades de uma língua de sujeito nulo prototípica, admitindo, por exemplo, leitura não referencial de sujeitos nulos de terceira pessoa, bem como o preenchimento da posição de sujeito por elementos não canônicos de interpretação dêitica/locativa (espacial e temporal). ${ }^{1}$

Pilati e Naves $(2011,2013)$ argumentam que a inovação na sintaxe do sujeito no $\mathrm{PB}$ está relacionada à perda do traço de referencialidade da terceira pessoa, em oposição à primeira e à segunda pessoa, em que esse traço é inerente. Trata-se, segundo as autoras, de um caso de cisão no sistema pronominal/flexional, em que a terceira pessoa no $\mathrm{PB}$ deixa de licenciar sujeitos nulos referenciais em contextos out-of-the-blue - cf. (1) -, diferentemente do que ocorre nas línguas de sujeito nulo canônicas - cf. dados do italiano em (2).

a. Ela fala italiano. [terceira pessoa - sujeito manifesto]

b. *Fala italiano. [terceira pessoa - sujeito nulo, out-of-the-blue].

a. Lui parla italiano. (RIZZI, 1982, p. 143)

b. Parla italiano.

Segundo Pilati e Naves $(2011,2013)$ e, posteriormente, Pilati, Naves e Salles (2017a, 2017b, 2017c), os contextos de licenciamento de sujeitos inovadores no PB apresentados de (I) a (IV) a seguir, podem ser analisados em termos dessa mudança nos traços formais da terceira pessoa, que, por sua vez, se deve a alterações no sistema pronominal/flexional, amplamente referidas na literatura gerativista e variacionista (DUARTE, 1995; GALVES, 2001; KATO e DUARTE, 2008; entre muitos outros).

\section{(I) ORDEM VERBO-SUJEITO COM INVERSÃO LOCATIVA}

Pilati (2006), ao analisar as propriedades sintáticas e semânticas das orações com ordem VS no PB, chega às seguintes conclusões: a) tais orações não são usadas em contextos de respostas a perguntas QU- com foco estreito - cf.

\footnotetext{
${ }^{1}$ O termo "inovador" não está, neste artigo, correlacionado à visão eurocêntrica da descrição linguística. Referimo-nos, antes, à diferenciação entre a sintaxe do sujeito no PB e a sintaxe das línguas de sujeito nulo canônicas (com as quais o termo "inovador" pretende criar um contraste). Portanto, não são as construções aqui analisadas inovadoras por si mesmas (ao contrário, em alguns casos, podem ser encontrados dados dessas construções em textos dos séculos XVIII e XIX, o que permite demonstrar que essa sintaxe tem origem em períodos anteriores, além do que construções como essas têm sido atestadas para outras línguas), mas é a análise unificada desses fenômenos em termos da sintaxe do sujeito que propicia sua designação como estruturas inovadoras.
} 
(3B'"); b) são licenciadas com mais frequência com verbos inacusativos, como atesta a maioria dos estudos variacionistas - cf. (4); c) podem ocorrer com verbos inergativos e transitivos - cf. (5).

(3) A: Quem dormiu/comeu o bolo?

(PILATI, 2006)

B: Foi a Bruna (que dormiu/que comeu o bolo).

B': A Bruna.

B": *Dormiu a Bruna. $/{ }^{*}$ Comeu o bolo a Bruna.

(4) Chegou a carta.

(PILATI, 2006)

(5) a. Toma posse o deputado.

(PILATI, 2002)

b. Ali dorme a Maria.

(PILATI, 2002)

c. E dançaram as duas.

(SCHERRE et al, 2007)

Para Pilati (2006, p. 199), os sujeitos nulos das orações com ordem VS do $\mathrm{PB}$ recebem uma interpretação dêitica/locativa (espacial ou temporal), como ilustrado a seguir.

a. Entrou Dante. [= Dante entrou (aqui)]

b. Dante entrou. [= Dante entrou (em algum lugar)]

a. Morreu Fellini. [= Fellini acabou de morrer $]$

b. Fellini morreu. [= Fellini morreu (há algum tempo)]

Outra evidência apresentada pela autora é a interpretação de orações em que o elemento dêitico manifesto na posição de sujeito - especificador do sintagma tempo, do inglês, temporal phrase (specTP) - da oração matriz controla sujeitos encaixados, comportando-se como sujeitos prototípicos - cf. (8).

a. As crianças $s_{i}$ dormem ali porque $e_{i}$ querem.

b. *Ali dormem as crianças $s_{i}$ porque $e_{i}$ querem.

c. $\mathrm{Ali}_{\mathrm{i}}$ dormem as crianças porque $\mathrm{e}_{\mathrm{i}}$ é mais quente. locativa.

Nesse sentido, a estrutura VS do PB é analisada como um tipo de inversão

\section{(II) Construções de tópico-sujeito}

Nas construções de tópico-sujeito do $\mathrm{PB}$, sintagmas com interpretação possessiva (9a), locativa (9b) ou benefactiva (9c) ocupam posição pré-verbal, supostamente associada ao sujeito, uma vez que se observa concordância com o verbo (10) - cf. Pontes (1986).

a. O carro furou o pneu.

b. A localidade não ocorreu nenhum problema

(PONTES, 1986)

c. O Fluminense faltou sorte no segundo tempo. 
(10) Os carros furaram o pneu.

Seguindo Pilati (2006), Pilati e Naves (2011, 2013) observam que o DP pré-verbal pode controlar um sujeito na oração encaixada, sendo possível a correferência entre o DP locativo, por hipótese na posição de sujeito da matriz, e o sujeito (nulo) na oração encaixada - cf. (11).

(11) a. A localidade ${ }_{i}$ não ocorreu nenhum problema porque pro $_{\mathrm{i}}$ é segura.

b. A Sarinha ${ }_{\mathrm{i}}$ tá nascendo dente porque pro $_{\mathrm{i}}$ tem seis meses de idade.

(III) construções com verbos transitivos e sujeito nulo arbitrário sem o pronome se

O PB também licencia sujeitos nulos arbitrários em construções como as de (12), em que se depreende ou identifica uma interpretação dêitica por meio de um locativo (espacial/temporal).

(12) a. Nos nossos dias, não usa mais saia.

(GALVES, 1987)

b. Vende frutas. [em uma placa]

c. Aqui faz conserto de roupas.

(PILATI;NAVES, 2013)

(PILATI;NAVES, 2013)

(IV) Construções com sujeitos não-argumentais

O quarto tipo de construção examinado por Pilati e Naves $(2011,2013)$ se caracteriza pela ocorrência de constituintes não-argumentais em posição de sujeito:

a. Brasília não chove há mais de 90 dias.

b. Londres tem prédios lindos.

(PILATI;NAVES, 2013)

(KATO;DUARTE, 2008)

As autoras argumentam que essas construções têm um correlato em que a posição de sujeito é nula e o sintagma locativo ocorre como um adjunto, tal como em $(14 a, b)$ :

(14) a. Não chove há mais de 90 dias em Brasília.

b. Tem prédios lindos em Londres.

Para Pilati e Naves $(2011,2013)$, esses quatro tipos de construções podem ser analisados como resultantes de um mesmo fenômeno linguístico no que se refere ao estatuto da categoria realizada na posição de sujeito, porque apresentam as seguintes semelhanças:

a) recebem interpretação dêitica, predominantemente locativa/temporal, não sendo licenciados fora desses contextos dêiticos; 
b) ocorrem com verbos na terceira pessoa;

c) elementos dêiticos ocupam posição típica de sujeito, mesmo quando não são argumentos dos predicados.

Considerando a possibilidade de analisar diferentes contextos de licenciamento do sujeito com base em uma hipótese unificada, este artigo tem duplo objetivo: (I) descrever e analisar o licenciamento de sujeitos inovadores (nulos e manifestos) no Português Brasileiro (PB) sob o ponto de vista sincrônico, considerando a proposta de que a realização do sujeito é crucialmente determinada pela perda do traço de referencialidade na terceira pessoa, o que resulta na cisão do sistema pronominal/flexional; (II) apresentar dados da diacronia do sujeito no PB, em comparação com o PE (cf. GRAVINA, 2014), como evidência para a cisão no paradigma pronominal/flexional do PB (cf. RABELO, 2010) e para a manifestação da ordem VS como inversão locativa.

$\mathrm{O}$ artigo está organizado nas seguintes seções, além desta introdução e das considerações finais: na seção 1, são apresentadas as mudanças no paradigma pronominal/flexional, em especial relacionadas à terceira pessoa, as quais consideramos deflagradoras das inovações na gramática do PB; na seção 2, são descritas as diferentes construções na gramática inovadora dos sujeitos do PB; na seção 3, é apresentada a proposta teórica para o comportamento de sujeitos nulos no $\mathrm{PB}$; na seção 4 , são descritos aspectos diacrônicos referentes à sintaxe do sujeito em PB como evidência para a proposta da cisão do sistema pronominal/flexional.

\section{AS MUDANÇAS NO PARADIGMA PRONOMINAL/FLEXIONAL DE TERCEIRA PESSOA NA GRAMÁTICA DO PB}

Conforme apresentado na introdução, para Pilati e Naves (2011, 2013), o licenciamento de estruturas aparentemente distintas, tais como a ordem VS, orações com sujeito indeterminado (manifesto ou nulo), orações de tópico-sujeito e construções com sujeitos não argumentais, pode estar relacionado a um mesmo fenômeno sintático, que é a cisão da categoria pronominal no $\mathrm{PB}$, em termos das propriedades relativas à $3^{\mathrm{a}}$ pessoa.

Buscando estabelecer uma relação entre as mudanças na flexão de terceira pessoa e o licenciamento desses quatro tipos de construções, Pilati e Naves (2013) baseiam-se em Alexiadou e Anagnostopoulou (1998), que defendem que o traço EPP se relaciona à checagem de um traço nominal $\mathrm{D}$ de Agr (CHOMSKY, 1995) e as línguas podem satisfazer EPP via movimento do verbo para $T$, desde que a morfologia verbal de concordância inclua esse traço nominal. ${ }^{2}$ Ainda segundo Alexiadou e Anagnostopoulou (1998), em línguas de sujeito nulo canônicas, a flexão manifesta um elemento nominal com traços D e phi capazes de checar o

\footnotetext{
${ }^{2}$ EPP: do inglês, Extended Projection Principle. Agr: do inglês, Agreement.
} 
traço EPP de T. Em contraposição, há línguas em que a morfologia verbal de concordância não contém o traço D e o traço EPP tem de ser satisfeito via concatenação de um DP em specTP. ${ }^{3}$

A proposta teórica de Pilati e Naves (2013) para o PB é a de que a morfologia verbal de terceira pessoa nessa língua, apesar de checar os traços phi de $T$, não contém o elemento nominal com traço D capaz de satisfazer EPP, ou seja, não pode checar a referencialidade da sentença, do que decorrem duas consequências: (I) necessidade de preenchimento da posição de sujeito no PB; (II) impossibilidade de referência autônoma. As autoras propõem que a terceira pessoa no PB se comporta como proforma - no sentido que Bhat (2004) atribui às formas pronominais de caráter dêitico -, por deixar de denotar a referência à terceira pessoa como participante do discurso.

Segundo Pilati e Naves (2013, p. 249), essa análise se distingue de outras em que se considera a existência de um T com traços-phi defectivos em PB (NUNES, 2008; RODRIGUES, 2004), pois as autoras apresentam um tratamento para as construções inovadoras do PB em termos do licenciamento de categorias codificadoras da referencialidade. Dessa análise, emergem as seguintes questões, que discutimos nas seções 2 e 3 , respectivamente:

a) como explicar a derivação de construções com diferentes tipos de verbo, ou seja, com diferentes estruturas argumentais?

b) como explicar a referência locativa das orações de sujeitos nulos?

\section{AS DIFERENTES ESTRUTURAS SINTÁTICAS NA GRAMÁTICA DOS SUJEITOS DO PB}

Pilati, Naves e Salles (2017a, p. 78), ao retomarem a questão relacionada às estruturas sintáticas formadas pelos diferentes tipos de verbo, propõem, seguindo Pilati e Naves (2013) e Naves, Pilati e Salles (2013), que as mudanças na natureza dos traços phi dos núcleos funcionais relevantes permitem o licenciamento da inversão locativa no $\mathrm{PB}$, bem como de sujeitos locativos (nulos

\footnotetext{
${ }^{3} \mathrm{Na}$ derivação da sentença, assumimos Chomsky (1995) para o licenciamento de traços formais, em que a projeção Agr é excluída. Na presente análise, o licenciamento do traço nominal $\mathrm{D}$ do sistema pronominal/flexional pode ser formalizado em termos das propriedades flexionais do verbo (de acordo com a proposta de Alexiadou e Anagnostopoulou, 1998), via movimento do verbo (desde que a morfologia de concordância inclua esse traço nominal), ou em termos de uma projeção phi que se incorpora em T - nos termos de Holmberg (2010) -, ou via concatenação de um DP em specTP. O tratamento aludido nessa implementação se assemelha ao proposto por Kato (1999), em artigo sobre o sujeito em línguas românicas.
} 
ou manifestos) e de elementos locativos (DPs ou pronomes/advérbios) na posição de sujeito. ${ }^{4}$

Para as autoras, os elementos locativos na posição de sujeito no PB podem ser expressos por dois tipos de categorias gramaticais, ambas satisfazendo o traço EPP: DPs plenos ou pronomes adverbiais, de um lado, e pronomes expletivos (nulos ou manifestos), de outro. Nesse sentido, as autoras ampliam a análise inicial e mostram que existem dois tipos de elementos locativos (nulos ou lexicais) em posição de sujeito no PB: (I) os de natureza argumental - cf. (15) - e os de natureza quasi-argumental/não-argumental - cf. (16) a (19). Ou seja, o tipo de elemento locativo está diretamente relacionado às propriedades do predicado, satisfazendo, em ambos os casos, a exigência de uma categoria dêitica na estrutura oracional. As representações arbóreas das quatro construções em discussão são apresentadas a seguir:

\section{(I) Construções de tópico-sujeito (verbos inacusativos)}

(15) $\left[\mathrm{CP}\left[\mathrm{TP}[\mathrm{DP} \text { As janelas }]_{\mathrm{k}}\left[\mathrm{T}\right.\right.\right.$, quebraram $\left[\mathrm{vP}_{\mathrm{i}}\left[\mathrm{DP}\right.\right.$ o vidro $\left.\left.\left.\left.\mathrm{t}_{\mathrm{k}}\right]\right]\right]\right]$

\section{(II) Construções com sujeitos arbitrários}

(16) Verbo transitivo:

a. [ст [тр Aqui [ $\mathrm{T}$, vende ${ }_{\mathrm{i}}\left[\mathrm{vP} \mathrm{t}_{\mathrm{i}}\right.$ fruta]]]]

b. [CP $\left[\mathrm{TP}_{\mathrm{P}}\right.$ pro $_{\text {Loc }}\left[\mathrm{T}\right.$, vende ${ }_{\mathrm{i}}\left[\mathrm{vP}_{\mathrm{i}}[\mathrm{DP}\right.$ fruta $\left.\left.\left.\left.]\right]\right]\right]\right]$ [em uma placa]

(17) Verbo inergativo:

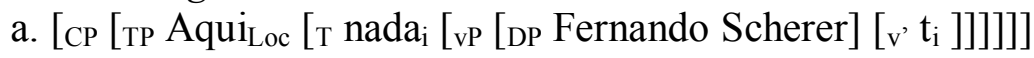

${ }^{4}$ Tendo em vista a adoção da proposta de Pilati e Naves (2013), e de Pilati et al. (2017), faz-se necessário explicitar o mecanismo de verificação dos traços formais de tempo/modo/aspecto, juntamente com os traços phi e de referencialidade em relação ao núcleo T. Nos termos da presente análise, os traços de tempo/modo/aspecto são interpretáveis e, portanto, não requerem verificação por um sistema de checagem. Em relação ao licenciamento dos traços phi e ao traço EPP, remetemos à nota 2. No que se refere aos outros modelos que envolvem a defectividade dos traços de $\mathrm{T}$, como os de Rodrigues (2004) e Nunes (2008), consideramos que essas hipóteses não dão conta das construções ilustradas neste estudo, exatamente porque não respondem às questões colocadas em (a) e (b) - a defectividade de T não daria conta de explicar os contrastes estabelecidos pelos tipos de verbos nem o papel do DP locativo.

${ }^{5}$ As autoras seguem Munhoz e Naves (2012), para quem, nas construções de tópicosujeito, o sujeito é realizado por uma categoria de caráter argumental, o que pressupõe que os verbos inacusativos sejam monoargumentais (cf. (15)) ou biargumentais (cf. Essa casa bate sol, em que o locativo é considerado um argumento interno). A derivação em (15), em que o DP o vidro e o DP as janelas são concatenados em estrutura de small clause projetando um DP, segue o proposto por Lunguinho (2006) e adotado por Munhoz e Naves (2012). 
b. * [Cр [тP Essas piscinas [T, nadam ${ }_{k}\left[\mathrm{vp}\left[\mathrm{DP}\right.\right.$ Fernando Scherer] [v $\left.\left.\left.\left.\left.\left.{ }^{\prime} \mathrm{t}_{\mathrm{i}}\right]\right]\right]\right]\right]\right]$

(III) Verbos com quasi-argumentos

(18) a. [СР [тP Essas cidades $\left[\mathrm{T}\right.$, chovem $_{\mathrm{i}}\left[\mathrm{vP}_{\mathrm{i}}\right.$ muito $\left.\left.]\right]\right]$

b. $\left[{ }_{\mathrm{CP}}\left[\mathrm{TP}\right.\right.$ Aqui $\left[\mathrm{T}\right.$, chove $\left.\left.\left.\left[\mathrm{vp}_{\mathrm{i}}\right]\right]\right]\right]$

(IV) Ordem vs (inversão locativa)

(19) $\left[\mathrm{CP}\left[\mathrm{C}\left[\mathrm{TP}\right.\right.\right.$ Aqui/pro $\operatorname{poc}_{\text {[T, }}$ [vP morreu Fellini $\left.\left.\left.\left.]\right]\right]\right]\right]$

\section{REFINANDO A EXPLICAÇÃO TEÓRICA PARA O COMPORTAMENTO DE SUJEITOS NULOS NO PB}

Conforme mencionado, no contexto de oração raiz, caso o sujeito seja um DP pleno ou um pronome lexical, a leitura do sujeito é referencial - cf. $\left(20^{\mathrm{a}}, \mathrm{b}\right)-$, sendo agramatical nos casos de sujeito nulo em contextos out-of-the-blue - cf. (20c). Entretanto, segundo Pilati e Naves (2011, 2013), com predicados transitivos, sujeitos nulos de terceira pessoa podem ocorrer com leitura arbitrária, com ou sem elementos pronominais/adverbiais em posição pré-verbal - cf. (21).

(20) a. Maria conserta roupas.

b. Ela conserta roupas.

c. ${ }^{*}$ Conserta roupas.

(21) a. Conserta roupas. [em uma placa]

b. Antigamente consertava sapato, hoje joga fora e compra outro.

Já nas orações encaixadas, pronomes nulos de terceira pessoa podem se referir ou ao sujeito da oração matriz, ou ao tópico da oração (MODESTO, 2004, 2008; NEGRÃO, 1999) - cf. (22). Entretanto, em dados como os em (23), a presença de um locativo na oração encaixada impede que se estabeleça uma relação de ligação entre pro e o sujeito da oração matriz, que recebe interpretação arbitrária, confirmando-se a análise de Naves, Pilati e Salles (2013) para os contextos de oração raiz, proposta anteriormente.

(22) a. João disse que $p r o_{\mathrm{i}}$ vende cachorro quente na praia.

b. A Maria $a_{\mathrm{i}}$, João $\mathrm{o}_{\mathrm{j}}$ disse que $p r o_{\mathrm{i} /{ }^{*} \mathrm{j}}$ vende cachorro quente na praia.

(23) a. João disse que $p r o_{\mathrm{i}}$ vende cachorro quente na praia. (KATO; DUARTE, 2008)

b. João $\mathrm{i}_{\mathrm{i}}$ disse que aqui* ${ }_{\mathrm{i} j \mathrm{j}}$ vende cachorro quente.

Concluímos que as diferenças no comportamento de pro em orações raízes/matrizes e encaixadas é apenas superficial, um efeito dos diferentes tipos 
de relações de ligação. Para explicar o funcionamento de pro de terceira pessoa do $\mathrm{PB}$, em orações raízes/matrizes e encaixadas, por meio de uma análise unificada, Pilati, Naves e Salles (2017c) retomam a hipótese da ausência do traço de referencialidade na terceira pessoa e estabelecem uma comparação das propriedades da morfologia flexional de terceira pessoa do PB com as do sistema flexional do chinês, língua que possui sujeitos nulos, apesar de não apresentar um sistema de concordância. ${ }^{6}$

Inicialmente, Pilati, Naves e Salles (2017c) retomam a análise de Huang (1984), segundo a qual os sujeitos nulos do chinês podem se referir ou ao sujeito da oração matriz, ou a um tópico saliente no discurso. De acordo com Huang (1984), quando o sujeito nulo em (24a), por exemplo, se refere ao sujeito da matriz, comporta-se como pro, mas quando se refere ao um tópico, comporta-se como uma variável - cf. (24b).

a. Zhangsan ${ }_{i}$ shuo [ $\mathrm{e}_{\mathrm{i} j \mathrm{j}}$ bu renshi Lisi ]. Zhangsan say not know Lisi 'Zhangsan said he didn't know Lisi.'/ 'Z.i disse que (ele ${ }_{i j}$ ) não conhece L.'

b. Zhangsan ${ }_{i}$ shuo [ Lisi bu renshi $e_{*_{i j}}$ ]. Zhangsan say Lisi not know 'Zhangsan said Lisi didn't know him.'/ 'Z.i disse que L. não conhece ele*ij.'

Huang (1984) argumenta que, se o sujeito nulo em (24a) é pro, então o sintagma nominal Zhangsan é o seu antecedente, uma vez que é o elemento nominal mais próximo do sujeito nulo. Entretanto, nada impede que o objeto nulo em (24b) seja analisado como uma variável. Como resultado, o sujeito nulo em (24a) pode se referir tanto ao sujeito da oração matriz (Zhangsan) ou a alguém cuja referência é identificável no discurso. Já o objeto nulo, em (24b), só pode se referir a um tópico no discurso. Sendo assim, o sujeito nulo em chinês possui um estatuto duplo: quando ligado a uma posição A é um pro, quando ligado a uma posição A' se comporta como uma variável. Huang (1989) observa que diferentes categorias vazias são de fato "alocategorias" de uma mesma categoria vazia (algo análogo a alomorfes de um mesmo morfema), cuja identidade pode ser determinada localmente.

Considerando a proposta de Huang (1984, 1989), Pilati, Naves e Salles (2017c) argumentam que a mesma ideia pode ser aplicada à flexão de terceira

\footnotetext{
${ }^{6}$ Comparações entre o PB e o chinês já foram feitas por outros autores. A diferença de nossa análise para as de Modesto (2004, 2008), por exemplo, é que consideramos que a comparação só pode ser feita para a terceira pessoa do singular, uma vez que a primeira e a segunda pessoas, embora também estejam em contexto de mudança, podem ser recuperadas pela morfologia verbal, por possuírem leitura referencial inerente. Remetemos o leitor interessado na comparação entre propostas correntes na literatura acerca da sintaxe do sujeito ao trabalho de Pilati, Naves e Salles (2017c), em que as autoras estabelecem um percurso cronológico e teórico, a fim de apresentar a discussão de acordo com os pressupostos teóricos e empíricos assumidos por diferentes pesquisadores desse tema.
} 
pessoa no PB, fazendo-se as adaptações necessárias em termos minimalistas. De acordo com as autoras, em contexto encaixado:

a) pro se comporta como um pronome quando está ligado a um antecedente independentemente marcado por papel temático, que o c-comanda e é realizado em posição $\mathrm{A}$ - cf. (25):

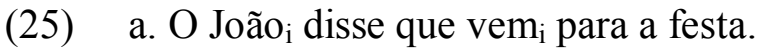

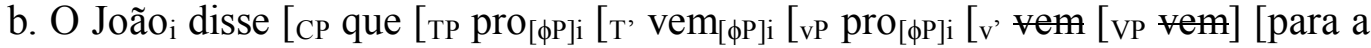
festa]]]]]]

Já em (26), a leitura arbitrária de pro surge pelo fato de que não existe um antecedente acessível. Por hipótese, a construção é licenciada na presença de um DP locativo (lexical ou nulo). Assumindo parcialmente a análise de Holmberg (2010), em relação à sintaxe de pro, propomos que o DP locativo em specTP valora o traço EPP, enquanto pro, realizado como uma categoria phi $\mathrm{P}$ em especificador de vP (specvP), valora os traços phi não-interpretáveis de $\mathrm{T}$, por incorporação ao núcleo T. Existe, portanto, uma identificação formal entre o DP locativo e o argumento externo, por meio do licenciamento do traço EPP e dos traços phi associados a T.

a. Aqui senta confortavelmente.

b. $\left[\right.$ се $\left[\mathrm{TP}\right.$ Aqui $\left.\left[\mathrm{T}, \mathrm{T}_{[\phi \mathrm{P}]} \operatorname{senta}\left[\mathrm{vP} \operatorname{pro}_{[\phi \mathrm{P}]}[\mathrm{v}, \operatorname{senta}[\mathrm{vP} \operatorname{senta}][\ldots]]\right]\right]\right]$

Essa operação é determinada pelas seguintes condições: a) a perda do SE indefinido (que está em distribuição complementar com o DP locativo em specTP); b) a identificação formal do argumento externo com um sintagma locativo (temporal/espacial), como Aqui em (26a), Essa casa/Esse carro nas construções de tópico-sujeito como Essa casa bate sol/Esse carro furou o pneu, Essas cidades em construções com predicados meteorológicos como Essas cidades chovem muito, ou um tipo de prosopopeia, como em Essa loja vende fruta. Essa análise se apoia no fato de que o traço de concordância (supostamente o de número) emerge nesse contexto, como ilustrado em (27).

(27) a. Essas lojas venderam muitos CDs.

b. Essas cidades chovem muito.

b) pro funciona como uma variável em contextos em que se liga a um elemento em posição A', como nas sentenças com tópico ou de pergunta e resposta.

(28) a. A Maria ${ }_{i}$, o João disse que vemi $_{\mathrm{i}}$ para a festa.

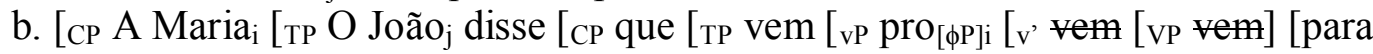
a festa]]]]]]]

Essa análise dá conta dos fatos contrastivos em (29a) e (29b), em que o sujeito nulo é um pro licenciado sob a relação de concordância acima descrita 
com um sintagma locativo em posição de sujeito e sob a ligação por um antecedente local que o c-comanda, respectivamente.

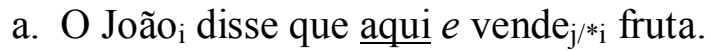
'John said that one sells fruits here'

b. O João disse que vende $_{i}$ fruta.

'John said that he sells fruits'

$\mathrm{Na}$ seção a seguir, apresentamos evidência diacrônica para a cisão pronominal postulada na presente análise, conforme discutido em Pilati, Naves e Salles (2017b), demonstrando a assimetria entre a primeira e a terceira pessoa no $\mathrm{PB}$, bem como o fato de que, desde o século XIX, a ordem verbo-sujeito no PB tem se configurado como inversão locativa, o que se confirma pelo importante papel dos locativos na primeira posição da sentença.

\section{ASPECTOS DIACRÔNICOS DA MUDANÇA NO PB: CISÃO E LOCATIVOS PRÉ- VERBAIS}

Seguindo Pilati, Naves e Salles (2017b), apresentamos a seguir o estudo comparativo-diacrônico feito por Gravina (2014), a respeito do uso do sujeito nulo e da inversão do sujeito no $\mathrm{PB}$ e no $\mathrm{PE}$, que tem por objetivo investigar a relação entre o sujeito nulo e a inversão do sujeito na diacronia. ${ }^{7}$ As tabelas a seguir, retiradas de Gravina (2014), ilustram os comportamentos distintos do PB e do PE.

Tabela 1: Distribuição dos sujeitos nulos pelas pessoas do discurso nos jornais brasileiros

\begin{tabular}{|l|c|c|c|}
\hline & $\begin{array}{c}\text { Número de Sujeitos } \\
\text { Nulos de primeira } \\
\text { pessoa }\end{array}$ & $\begin{array}{c}\text { Número de Sujeitos } \\
\text { Nulos de segunda } \\
\text { pessoa }\end{array}$ & $\begin{array}{c}\text { Número de Sujeitos } \\
\text { Nulos de terceira } \\
\text { pessoa }\end{array}$ \\
\hline Recreador Mineiro & $306 / 688-45 \%$ & $48 / 688-7 \%$ & $334 / 688-48 \%$ \\
\hline Jornal Mineiro & $304 / 419-72 \%$ & $10 / 419-3 \%$ & $105 / 419-25 \%$ \\
\hline $\begin{array}{l}\text { Tribuna de Ouro } \\
\text { Preto }\end{array}$ & $237 / 312-76 \%$ & $19 / 312-7 \%$ & $56 / 312-17 \%$ \\
\hline
\end{tabular}

Fonte: GRAVINA (2014)

${ }^{7}$ O corpus foi extraído de jornais que circularam em Ouro Preto/Brasil - O Recreador Mineiro (1845-1848); O Jornal Mineiro (1897-1900); Tribuna de Ouro Preto (19451948) - e em Évora/Portugal - A Illustração luzo-brasileira (1856-1858); $O$ Manuelinho de Évora (1890-1898); Notícias de Évora (1945-1948). 
Tabela 2: Distribuição dos sujeitos nulos pelas pessoas do discurso nos jornais portugueses

\begin{tabular}{|l|c|c|c|}
\hline & $\begin{array}{c}\text { Número de Sujeitos } \\
\text { Nulos de primeira } \\
\text { pessoa }\end{array}$ & $\begin{array}{c}\text { Número de Sujeitos } \\
\text { Nulos de segunda } \\
\text { pessoa }\end{array}$ & $\begin{array}{c}\text { Número de Sujeitos } \\
\text { Nulos de terceira } \\
\text { pessoa }\end{array}$ \\
\hline $\begin{array}{l}\text { Ilustração luzo- } \\
\text { brasileira }\end{array}$ & $255 / 881-29 \%$ & $88 / 881-10 \%$ & $538 / 881-61 \%$ \\
\hline $\begin{array}{l}\text { Manuelinho de } \\
\text { Évora }\end{array}$ & $163 / 710-23 \%$ & $57 / 710-8 \%$ & $490 / 710-69 \%$ \\
\hline Notícias de Évora & $166 / 615-27 \%$ & $74 / 615-12 \%$ & $375 / 615-61 \%$ \\
\hline
\end{tabular}

\section{Fonte: GRAVINA (2014)}

O maior número de sujeitos nulos de primeira pessoa em relação aos de terceira pessoa nos dados $\mathrm{PB}$, quando comparados aos do PE, no total de ocorrências de sujeito em cada jornal, é inquestionável. Por sua vez, os baixos índices de sujeito nulo de primeira pessoa no PE podem ser associados a fatores independentes. Pilati, Naves e Salles (2017b) observam que a distinção entre as pessoas está ligada à mudança no sistema pronominal do $\mathrm{PB}$, o que reforça a hipótese da cisão pronominal. Prova disso é o comportamento assimétrico do PB na distribuição da primeira e da terceira pessoa, não observado no PE, uma língua que não apresenta cisão de pessoa no sistema pronominal/flexional.

Em relação à presença de locativos na posição de sujeito no $\mathrm{PB}$, o estudo de Gravina (2014) traz resultados interessantes. Quanto ao percentual de licenciamento de SV e VS, não há grande diferença nos dados dos periódicos do Brasil e de Portugal. Analisando, porém, a relação entre a distribuição de elementos adverbiais e o tipo de verbo, observamos uma diferença nos contextos em que orações com ordem VS ocorrem nos dados do PE e do PB: em PB, os maiores índices de ordem VS são na configuração XVS (intransitivos), enquanto em PE a maior ocorrência da ordem VS é em contextos XOVS (transitivos).

Tabela 3: Proporção de cada tipo de inversão no português brasileiro

\begin{tabular}{|c|c|c|c|c|c|c|c|}
\hline & VS & XVS & VOS & XVOS & OVS & XOVS & VXS \\
\hline $\begin{array}{l}\text { Recreador } \\
\text { Mineiro } \\
\text { (1845 a 1848) }\end{array}$ & $\begin{array}{c}22 / 72 \\
30 \%\end{array}$ & $\begin{array}{c}28 / 72 \\
39 \%\end{array}$ & $\begin{array}{l}9 / 72 \\
12 \%\end{array}$ & $\begin{array}{c}1 \\
0,5 \%\end{array}$ & $\begin{array}{l}7 / 72 \\
10 \%\end{array}$ & $\begin{array}{c}4 / 72 \\
5 \%\end{array}$ & $\begin{array}{l}3 / 72 \\
3,5 \%\end{array}$ \\
\hline $\begin{array}{l}\text { Jornal Mineiro } \\
(1887 \text { a 1900) }\end{array}$ & $\begin{array}{c}15 / 83 \\
18 \%\end{array}$ & $\begin{array}{c}43 / 83 \\
52 \%\end{array}$ & $\begin{array}{c}10 / 83 \\
12,5 \%\end{array}$ & $\begin{array}{c}2 / 83 \\
2 \%\end{array}$ & $\begin{array}{c}5 / 83 \\
6 \%\end{array}$ & $\begin{array}{l}8 / 83 \\
9,5 \%\end{array}$ & $\begin{array}{c}0 / 83 \\
0 \%\end{array}$ \\
\hline $\begin{array}{l}\text { Tribuna de } \\
\text { Ouro Preto } \\
\text { (1945 a 1948) }\end{array}$ & $\begin{array}{c}13 / 61 \\
21 \%\end{array}$ & $\begin{array}{c}20 / 61 \\
33 \%\end{array}$ & $\begin{array}{c}22 / 61 \\
37 \%\end{array}$ & $\begin{array}{c}3 / 61 \\
4 \%\end{array}$ & $\begin{array}{c}0 / 61 \\
0 \%\end{array}$ & $\begin{array}{l}1 / 61 \\
1,5 \%\end{array}$ & $\begin{array}{c}2 / 61 \\
3,5\end{array}$ \\
\hline
\end{tabular}

Fonte: GRAVINA (2014) 
Tabela 4: Proporção de cada tipo de inversão no português europeu

\begin{tabular}{|c|c|c|c|c|c|c|c|}
\hline & VS & XVS & VOS & XVOS & OVS & Xovs & VXS \\
\hline Illustração & $27 / 91$ & $8 / 91$ & 7/91 & $10 / 91$ & 2/91 & $34 / 91$ & $3 / 91$ \\
\hline luso-brasileira & $30,5 \%$ & $9 \%$ & $7,5 \%$ & $11 \%$ & $2 \%$ & $37 \%$ & $3 \%$ \\
\hline $\begin{array}{l}\text { Manuelinho de } \\
\text { Évora }\end{array}$ & $\begin{array}{c}24 / 100 \\
24 \%\end{array}$ & $\begin{array}{c}4 / 100 \\
4 \%\end{array}$ & $\begin{array}{c}6 / 100 \\
6 \%\end{array}$ & $\begin{array}{c}8 / 100 \\
8 \%\end{array}$ & $\begin{array}{c}4 / 100 \\
4 \%\end{array}$ & $\begin{array}{c}48 / 100 \\
48 \%\end{array}$ & $\begin{array}{c}6 / 100 \\
6 \%\end{array}$ \\
\hline $\begin{array}{l}\text { Notícias de } \\
\text { Évora }\end{array}$ & $\begin{array}{l}17 / 119 \\
14,5 \%\end{array}$ & $\begin{array}{l}3 / 116 \\
2,5 \%\end{array}$ & $\begin{array}{c}12 / 119 \\
10 \%\end{array}$ & $\begin{array}{c}13 / 119 \\
11 \%\end{array}$ & $\begin{array}{c}4 / 119 \\
3 \%\end{array}$ & $\begin{array}{c}63 / 119 \\
53 \%\end{array}$ & $\begin{array}{c}7 / 119 \\
6 \%\end{array}$ \\
\hline
\end{tabular}

Fonte: GRAVINA (2014)

Em suma, ao trazer dados da diacronia do PB e do PE, identificamos evidências para o surgimento da cisão pronominal. Nossa interpretação dos dados das tabelas de 1 a 4, de Gravina (2014), é que eles revelam não só a tendência a preencher os sujeitos no PB, confirmando-se as previsões de Tarallo (1983) e Duarte (1993), mas também que o sistema pronominal do PE apresenta propriedades distintas do sistema pronominal do $\mathrm{PB}$, principalmente no que se refere aos diferentes comportamentos das pessoas do discurso. Em particular, destacamos a estabilidade nos percentuais de sujeito nulo tanto de primeira quanto de terceira pessoa no $\mathrm{PE}$, diferentemente do $\mathrm{PB}$, em que se verifica o decréscimo na incidência de sujeitos nulos de terceira pessoa, em oposição aos de primeira pessoa, em que o índice de sujeito nulo aumenta. Percebemos, também, que os locativos são elementos sintáticos relevantes para o licenciamento da ordem VS no PB, na configuração XVS, evidenciando-se a importância dos sintagmas locativos na gramática do PB para dar sustentação à análise em termos da inversão locativa (PILATI, 2006).

\section{CONSIDERAÇÕES FINAIS}

No presente artigo, apresentamos o percurso das análises desenvolvidas por Pilati e Naves $(2011,2013)$ e por Pilati, Naves e Salles (2017a, 2017b, 2017c) para o licenciamento de sujeitos inovadores, nulos e manifestos no PB.

Por meio da hipótese da cisão do sistema pronominal/flexional, desenvolvemos uma análise unificada das construções sintáticas do PB mencionadas neste trabalho, contemplando uma explicação para a ocorrência de elementos locativos em posição de sujeito.

Observamos que, no PB, no período de tempo investigado por Gravina (2014), as taxas de sujeito nulo são instáveis não só em relação à diminuição de sujeitos nulos de terceira pessoa $(48 \%, 25 \%$ e $17 \%)$, mas também em relação às taxas de sujeito de primeira pessoa, que demonstram um crescimento significativo $(45 \%, 72 \%$ e $76 \%)$. 
Argumentamos que os fatos contrastivos no comportamento da primeira e da terceira pessoa fornecem evidência diacrônica para a cisão no sistema flexional/ pronominal do PB, particularmente no que se refere à ausência de propriedades referenciais na terceira pessoa. Dessa cisão decorrem as propriedades inovadoras na sintaxe do sujeito no $\mathrm{PB}$, o que permite unificar a análise em relação às seguintes construções do $\mathrm{PB}$ (não encontradas no $\mathrm{PE}$ ): (a) ordem VS com inversão locativa; (b) construções de tópico-sujeito; (c) construções de sujeito nulo indeterminado sem o pronome SE; (d) construções com DP locativos não-argumentais na posição de sujeito.

\section{REFERÊNCIAS}

AlEXIADOU, A.; ANAGNOSTOPOUlOU, E. Parametrizing Agr: word order, verb-movement and EPP-checking. Natural Language and Linguistic Theory. n. $16(3), 1998$, p. 491-539.

BERLINCK, R. et al. Predicação. In: KATO, NASCIMENTO, M. M. do. (Orgs.). Gramática do Português Culto Falado no Brasil. Campinas: SP, 2009, p. 97-188.

BHAT, D. N. S. Pronouns. Oxford: Oxford University Press, 2004.

CHOMSKY, N. Lectures on Government and Binding. Dordrecht: Foris, 1981.

CHOMSKY, N. The Minimalist Program. Cambridge, MA: The MIT Press, 1995.

DUARTE, M. E. L. Do pronome nulo ao pronome pleno: a trajetória do sujeito nulo no português do Brasil. In: ROBERTS, I.; KATO, M. (Orgs.). Português Brasileiro: Uma Viagem Diacrônica. Campinas: Unicamp, 1993, p. 107-128.

DUARTE, M. E. L. A perda do princípio 'Evite Pronome' no português do Brasil. Campinas, SP: 1995. 151f. Tese de Doutorado em Linguística. Campinas: Unicamp.

GALVES, C. A sintaxe do português brasileiro. Ensaios de Linguística. n. 13, 1987, p. 31-49.

GALVES, C. Ensaio sobre as Gramáticas do Português. Campinas: Editora da Unicamp, 2001.

GRAVINA, A. Sujeito nulo e ordem VS no português brasileiro: um estudo diacrônico comparativo baseado em corpus. Campinas, SP: 2014. 251f. Tese de Doutorado em Linguística. Campinas: UNICAMP. 
HOLMBERG, A. The null generic subject pronoun in Finnish. In: Biberauer, T.; HOLMBERG, A.; ROBERTS, I.; SHEENAN, M. (eds.) Parametric Variation: Null Subjects in Minimalist Theory. Cambridge: Cambridge University Press, 2010, p. 200-230.

HUANG, C.-T. J. On the distribution and reference of empty pronouns. Linguistic Inquiry. n. 15, 1984, p. 531-574.

HUANG, C.-T. J. Pro-drop in Chinese: a generalized control theory. In: JAEGGLI, O.; SAFIR, K. (eds.) The Null Subject Parameter. Dordrecht, Boston e London: Kluwer, 1989, p. 185-214.

KATO, M. Strong and weak pronominals in the null subject parameter. Probus. n. 11, 1999, p. 1-37.

KATO, M.; DUARTE, M. E. L. Mudança paramétrica e orientação para o discurso. Trabalho apresentado no XXIV Encontro Nacional da Associação Portuguesa de Linguística, Braga, 2008.

LUNGUINHO, M. V. da S. Partição de constituintes no português brasileiro: características sintáticas. In: Silva, D. E. G. da. (Org.) Língua, Gramática e Discurso. Goiânia: Cânone Editorial, 2006, p. 133-147.

MODESTO, M. Sujeitos nulos em línguas de tópico proeminente. Revista da ABRALIN. n. 3, 2004, p. 119-145.

MODESTO, M. Null subjects in Brazilian Portuguese and Finnish: they are not derived by movement. In: DAVIES, W.; DUBINSKY, S. (Eds.). New horizons in the Analysis of Control and Raising. Dordrecht: Springer, 2008, p. 231-248.

MUNHOZ, A. T.; NAVES, R. Construções de Tópico-Sujeito: uma proposta em termos de estrutura argumental e de transferência de traços de C. Signum. Estudos de Linguagem. n. 15, 2012, p. 245-265.

NAVES, R.; PILATI, E. As cidades da Amazônia chovem muito: non-thematic subjects and the properties of Infl in Brazilian Portuguese. Trabalho apresentado em Portuguese Linguistics in the United States Conference. Georgia: UGA, 2013.

NEGRÃO, E. V. O Português Brasileiro: uma língua voltada para o discurso. São Paulo, SP: 1999. 225f. Tese de Livre Docência em Linguística. São Paulo: Universidade de São Paulo.

NUNES, J. Inherent Case as a licensing condition for A-movement: the case of hyper-raising in Brazilian Portuguese. Journal of Portuguese Linguistics. n. 7(2), 2008, p. 83-108. 
PILATI, E. Sobre a ordem verbo-sujeito no português do Brasil. Brasília, DF: 2002. Dissertação de Mestrado em Linguística, Brasília: Universidade de Brasília.

PILATI, Eloisa. Aspectos sintáticos e semânticos da ordem verbo-sujeito no português. Brasília, DF: 2006. 242f. Tese de Doutorado em Linguística, Brasília: Universidade de Brasília.

PILATI, E.; NAVES, R. “A hipótese do sistema de transferência de traços de T para $\mathrm{C}$ e o licenciamento da posição de sujeito no português brasileiro". Trabalho apresentado no VII Congresso Internacional da ABRALIN, Curitiba-PR, 2011.

PILATI, E.; NAVES, R. Desenvolvendo a hipótese da cisão da categoria pronominal no português brasileiro. In: MOURA, D.; SIBALDO, M. (Eds.) Estudos e Pesquisas em Teoria da Gramática. Maceió: EDUFAL, 2013, p. 233253.

PILATI, E.; NAVES, R.; SALLES, H. Locative DPs and deictic adverbial pronouns in subject position in Brazilian Portuguese. In: FERNÁNDEZSORIANO, O.; CASTROVIEJO MIRÓ, E.; PÉREZ-JIMÉNEZ, I. (Eds.). Boundaries, Phases and Interfaces: Case studies in honor of Violeta Demonte. Amsterdam: John Benjamins, 2017a, p. 64-84.

PILATI, E.; NAVES, R.; SALLES, H. A cisão no sistema pronominal do português brasileiro: evidências na diacronia. In: PILATI, E.; SALLES, H. L.; NAVES, R. (Orgs.) Novos Olhares para a Gramática do Português Brasileiro. Campinas (SP): Pontes, 2017b, p. 151-169.

PILATI, E.; NAVES, R.; SALLES, H. On the syntax of subjects in Brazilian Portuguese: using the "split" pronominal system as the basis for an alternative analysis. Diadorim. n. 19 (especial), 2017c, p. 99-139.

PONTES, E. Sujeito: Da Sintaxe ao Discurso. São Paulo: Ática; Brasília: Instituto Nacional do Livro, Fundação Nacional Pró-Memória, 1986.

RABELO, P. Argumentos (EPP) Nulos no Português do Brasil em Contextos Oracionais Finitos e Infinitivos. Brasília, DF: 2010. 216f. Tese de Doutorado em Linguística. Brasília: Universidade de Brasília.

RIZZI, L. Issues in Italian Syntax. Dordrecht: Foris, 1982.

RODRIGUES, C. Impoverished Morphology and A-movement out of Casedomains. College Park, Maryland: 2004. 388f. Tese de Doutorado em Linguística. College Park: University of Maryland. 
SCHERRE, M. M.; NARO, A.; CARDOSO, C. O papel do tipo de verbo na concordância verbal no Português Brasileiro. DELTA. n. 23 (especial), 2007, p. 283-317.

TARALLO, F. Relativization Strategies in Brazilian Portuguese. Filadélfia, PA: 1983. 269f. Doctoral Thesis. University of Pennsylvania.

Eloisa Nascimento Silva Pilati EloisaPilati@gmail.com

Rozana Reigota Naves RozanaRN@hotmail.com

Heloisa Maria Moreira Lima Salles HeloisaSalles@gmail.com

Recebido em: 15 jan. 2018

Aceito em: 15 mar. 2018

Publicado em: 1 out. 2018 